\title{
Case Report \\ Staphylococcal Toxic Shock Syndrome Complicating Influenza A Infection in a Young Child
}

\author{
Timothy R. Peters, Dudley E. Hammon, Rima J. Jarrah, Elizabeth L. Palavecino, \\ Elizabeth S. Blakeney, and Katherine A. Poehling
}

The Departments of Pediatrics (TRP, DEH, ESB, KAP), Pediatric Critical Care (RJJ), and Pathology (ELP), Wake Forest School of Medicine, Medical Center Boulevard, Winston-Salem, NC 27157, USA

Correspondence should be addressed to Timothy R. Peters, tpeters@wfubmc.edu

Received 9 March 2011; Accepted 19 April 2011

Academic Editors: H. Hemilä and L. Molinos

Copyright ( $\odot 2011$ Timothy R. Peters et al. This is an open access article distributed under the Creative Commons Attribution License, which permits unrestricted use, distribution, and reproduction in any medium, provided the original work is properly cited.

Toxic shock syndrome (TSS) is a potentially lethal but rare complication of influenza infection. We report a case of TSS and influenza A infection in a 5-year-old boy without respiratory symptoms, in whom tracheal aspirate bacterial culture grew a toxinproducing strain of Staphylococcus aureus. Bacterial culture of the upper respiratory tract should be considered in patients with influenza-associated toxic shock syndrome.

\section{Introduction}

Staphylococcus aureus pneumonia is a well-known complication of influenza, and antibacterial drug resistance has increasingly complicated the management of staphylococcal disease [1]. TSS is life-threatening and may result from upper respiratory tract infection by toxin-elaborating strains of Staphylococcus aureus [2]. There have been rare reports of staphylococcal TSS complicating influenza disease in children, although in most of these cases isolation of the bacterial strain was not achieved [2-7]. Rates of TSS occurrence in patients with influenza disease are not known. We report a case of influenza complicated by TSS, in which tracheal aspirate culture yielded TSST-1-producing Staphylococcus aureus.

\section{Case Report}

A five-year-old boy was seen in the emergency department with complaints of fever, malaise, and myalgias. He was well until the previous day when he developed fever to 38.8 degrees, chills, and bilateral leg and foot pain without joint swelling. Earlier that day he was seen by his pediatrician who performed influenza rapid antigen testing which was positive for influenza $A$, and the patient was started on oseltamivir and amantadine in concordance with CDC recommendations for seasonal influenza A in February 2009. His symptoms of fever, malaise, and myalgias worsened through the day despite antiviral medications, and he presented to the emergency department for further evaluation. He did not have any respiratory symptoms-no cough, nasal congestion, or shortness of breath. He had no prior hospitalizations or surgeries. He had a history of asthma that was well controlled with montelukast and fluticasone propionate oral inhaler, and he had received the seasonal intramuscular influenza vaccine 5 months before admission and was completely vaccinated, having received two doses of influenza vaccine in a prior season.

On physical exam he appeared alert with a normal mental status. He was afebrile and tachypneic with a respiratory rate of 36 and otherwise normal vital signs. Pulse oximetry showed $94 \%$ oxygen saturation on room air. His chest was clear to auscultation without crackles or wheezes, and the physical examination was otherwise normal. Laboratory evaluation at admission showed WBC $9,500 / \mathrm{mm}^{3}$ with $63 \%$ granulocytes, $27 \%$ bands, $7 \%$ lymphocytes, and $3 \%$ monocytes, $136,000 / \mathrm{mm}^{3}$ and platelet count. The complete metabolic profile was normal except for serum bicarbonate of $18 \mathrm{mmol} / \mathrm{L}$ and total albumin level of $3.6 \mathrm{~g} / \mathrm{dL}$. Blood culture was sent and ultimately showed 
no growth. Nasopharyngeal swab for influenza detection was positive for influenza A by polymerase chain reaction assay. Chest radiograph showed only mild peribronchial thickening consistent with viral respiratory illness. He was admitted to the hospital for observation and continued antiviral therapy.

Within 12 hours of admission the patient developed diffuse erythroderma, conjunctival injection, and hypotension. Blood culture was repeated and ultimately showed no growth. Throat culture and rapid testing for Group A Streptococcus were negative. Aggressive fluid resuscitation and antibacterial therapy with vancomycin, clindamycin, and ceftriaxone was promptly initiated. The patient became lethargic and was transferred to the pediatric intensive care unit where he was treated with vasopressors for hypotension. Approximately 8 hours after antibiotics were started the patient was intubated for airway management due to shock and lethargy. At the time of intubation there were no airway abnormalities suggestive of bacterial tracheitis, and a tracheal aspirate was obtained.

Gram stain of this tracheal aspirate showed 2+ white blood cells and 1+ Gram-positive cocci. That sample grew $3+$ Staphylococcus aureus that was susceptible to oxacillin and cefazolin but resistant to penicillin, erythromycin, and clindamycin ( $D$-test positive). Antibacterial therapy was changed to intravenous oxacillin on hospital day 3. This isolate of Staphylococcus aureus was tested in the clinical microbiology laboratory and found to elaborate toxic shock syndrome toxin type 1 (TSST-1) by reverse passive latex agglutination.

Within 24 hours after pediatric intensive care unit admission the patient was successfully extubated, with marked improvement in blood pressure, mental status, and erythroderma. On hospital day 4 he was released from the pediatric intensive care unit to the general pediatric ward, and he was discharged home on hospital day 6 to complete 14 days of antibiotic therapy with oral cephalexin. He recovered completely.

\section{Discussion}

The decision to obtain a bacterial tracheal aspirate culture from our patient with influenza and suspected TSS aided greatly in his management, providing valuable drug susceptibility information and ultimately confirmation of TSST1 production in the isolated Staphylococcus aureus strain. The diagnosis of Staphylococcus aureus TSS is made using clinical case definition and does not depend on isolation of the pathogenic organism [8]. Laboratory isolation of pathogenic Staphylococcus aureus strains from patients with TSS is difficult. Blood culture is positive in fewer than $5 \%$ of patients with Staphylococcus aureus TSS [8]. However, the clinical value of Staphylococcus aureus strain isolation and drug susceptibility testing increases with increasing rates of antibacterial resistance. Methicillin-resistant Staphylococcus aureus is increasingly recognized as an important cause of TSS in children and adults [9].

Bacterial culture of tracheal aspirates at intubation is not routine when bacterial tracheitis is not suspected, and the appearance of the trachea was unremarkable at intubation in this patient. Indeed, bacterial cultures of the upper respiratory tract are of limited clinical usefulness and are seldom performed even in patients with probable bacterial sinusitis, given the high likelihood of polymicrobial infection, and the frequent isolation of nonpathogenic colonizing bacteria.

This interesting case demonstrates that culture of the upper respiratory tract may have special utility in cases of influenza-associated TSS. There are numerous reports of TSS associated with staphylococcus present in the nasopharynx, sinuses, pharynx, and trachea $[2,3,10]$. The performance characteristics (such as sensitivity and negative predictive value) of tracheal aspirate bacterial culture in cases of influenza-associated TSS are completely unknown. This is also true for culture of other upper respiratory sites including nasopharynx and throat. Recovery of colonizing bacterial strains would be expected, especially when bacterial tracheitis is not suspected. We propose that in suspected TSS, bacterial culture of the upper respiratory tract should be considered despite these limitations, with the specific goal of isolating Staphylococcus aureus for laboratory analysis.

In this patient with influenza infection followed by TSS, culture of the upper respiratory tract grew toxin-elaborating Staphylococcus aureus, and antibacterial susceptibility studies were tremendously useful in his subsequent management. Bacterial culture of the upper respiratory tract should be considered when TSS is suspected in children with influenza.

\section{Funding}

This work was supported in part by NIH R01 AI079226 for K. A. Poehling and T. R. Peters.

\section{References}

[1] A. J. Kallen, C. Reed, M. Patton, K. E. Arnold, L. Finelli, and J. Hageman, "Staphylococcus aureus community-onset pneumonia in patients admitted to children's hospitals during autumn and winter of 2006-2007," Epidemiology and Infection, vol. 138, pp. 666-672, 2010.

[2] R. W. Tolan Jr., "Toxic shock syndrome complicating influenza A in a child: case report and review," Clinical Infectious Diseases, vol. 17, pp. 43-45, 1993.

[3] K. L. MacDonald, M. T. Osterholm, C. W. Hedberg et al., "Toxic shock syndrome. A newly recognized complication of influenza and influenzalike illness," Journal of the American Medical Association, vol. 257, pp. 1053-1058, 1987.

[4] S. J. Sperber and J. B. Francis, "Toxic shock syndrome during an influenza outbreak," Journal of the American Medical Association, vol. 257, pp. 1086-1087, 1987.

[5] E. E. Conway Jr., R. S. Haber, J. Gumprecht, and L. P. Singer, "Toxic shock syndrome following influenza A in a child," Critical Care Medicine, vol. 19, pp. 123-125, 1991.

[6] M. L. Sion, A. I. Hatzitolios, E. N. Toulis, K. D. Mikoudi, and G. N. Ziakas, "Toxic shock syndrome complicating influenza A infection: a two-case report with one case of bacteremia and endocarditis," Journal of Intensive Care Medicine, vol. 27, p. 443, 2001.

[7] G. C. Prechter and A. K. Gerhard, "Postinfluenza toxic shock syndrome," Chest, vol. 95, pp. 1153-1154, 1989. 
[8] American Academy of Pediatrics, "Staphylococcal infections," in Red Book: 2009 Report of the Committee on Infectious Diseases, L. K. Pickering, C. J. Baker, D. W. Kimberlin, and S. S. Long, Eds., pp. 601-615, American Academy of Pediatrics, Elk Grove Village, Ill, USA, 2009.

[9] G. Durand, M. Bes, H. Meugnier et al., "Detection of new methicillin-resistant Staphylococcus aureus clones containing the toxic shock syndrome toxin 1 gene responsible for hospital- and community-acquired infections in France," Journal of Clinical Microbiology, vol. 44, pp. 847-853, 2006.

[10] J. K. Todd, "Toxic shock syndrome, Staphylococcus aureus, and influenza," Journal of the American Medical Association, vol. 257, pp. 3070-3071, 1987. 


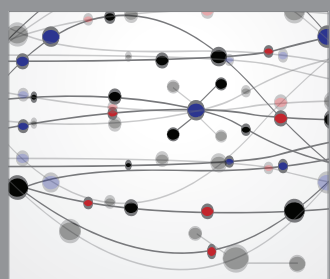

The Scientific World Journal
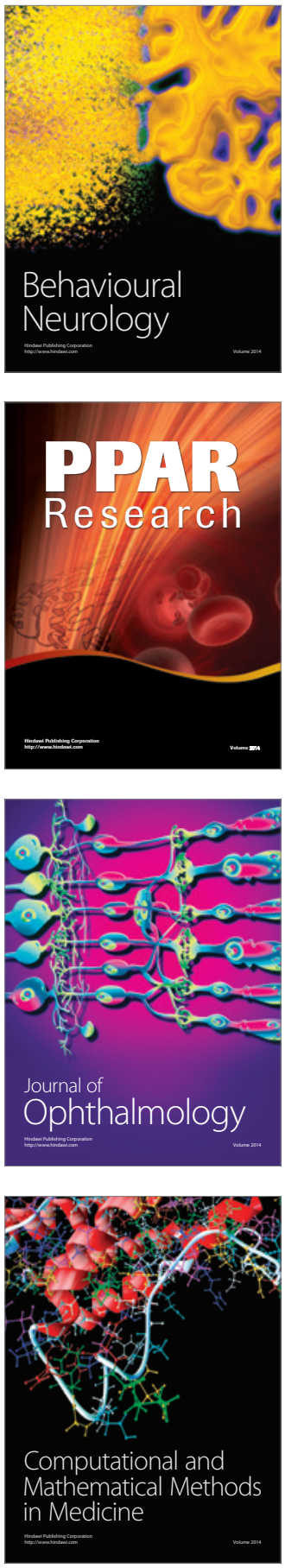

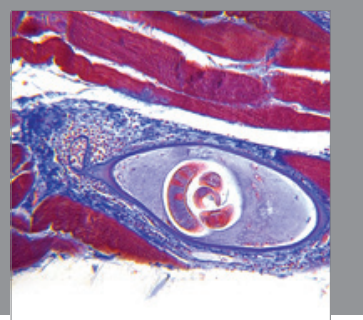

Gastroenterology

Research and Practice
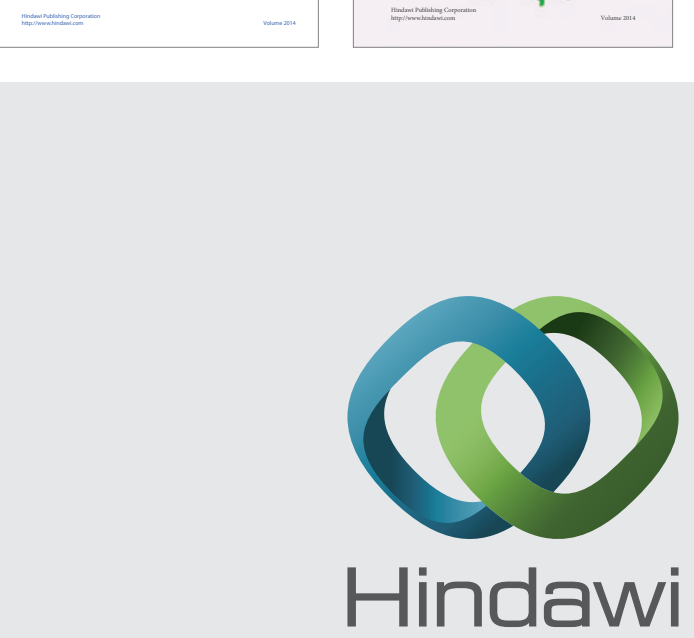

Submit your manuscripts at

http://www.hindawi.com
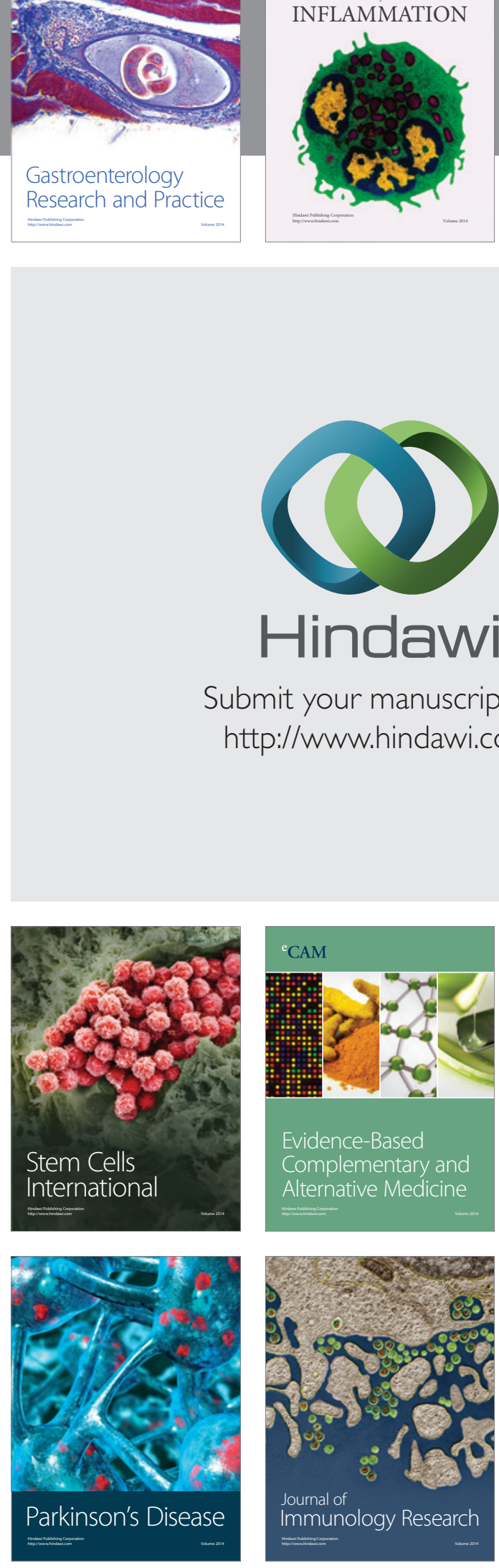

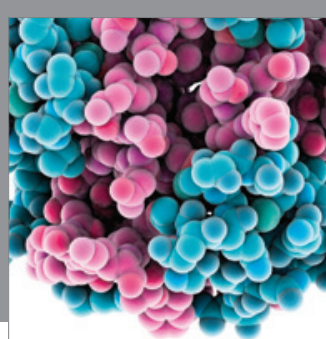

Diabetes Research
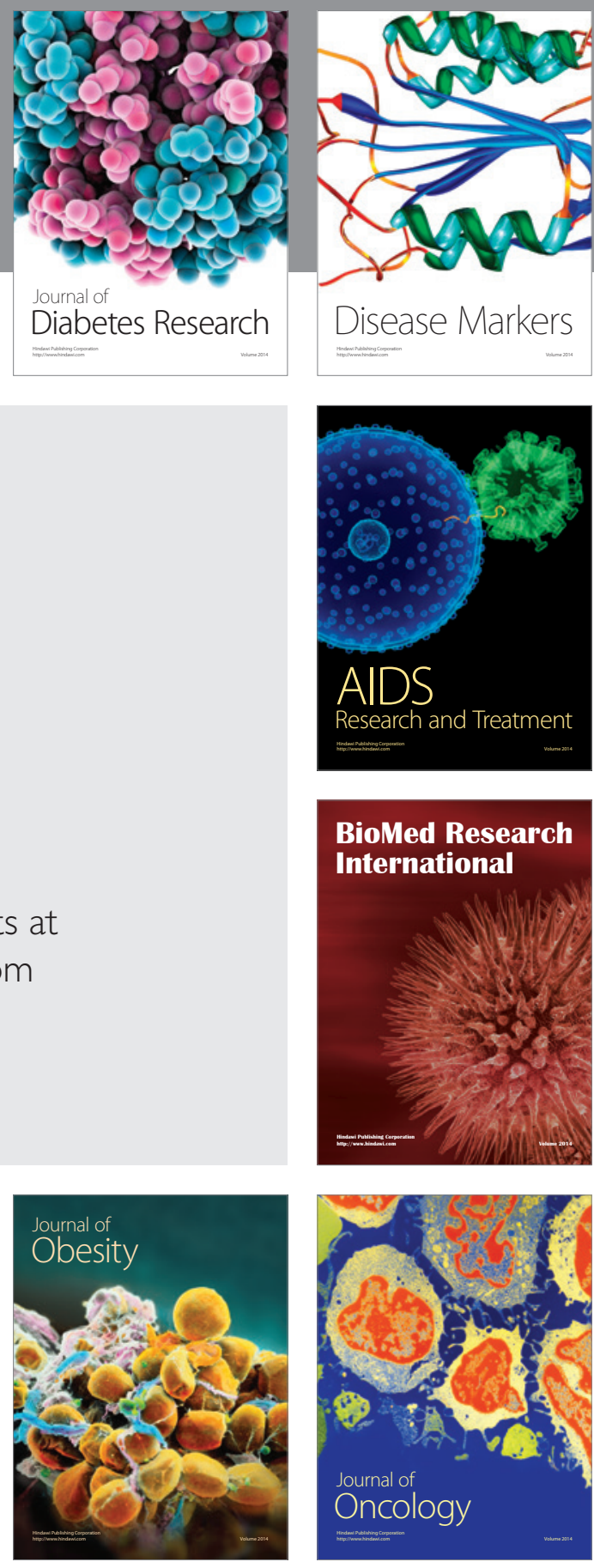

Disease Markers

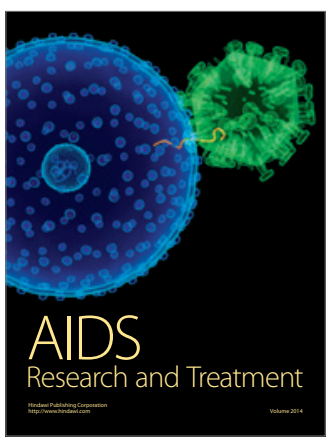

BioMed Research

International
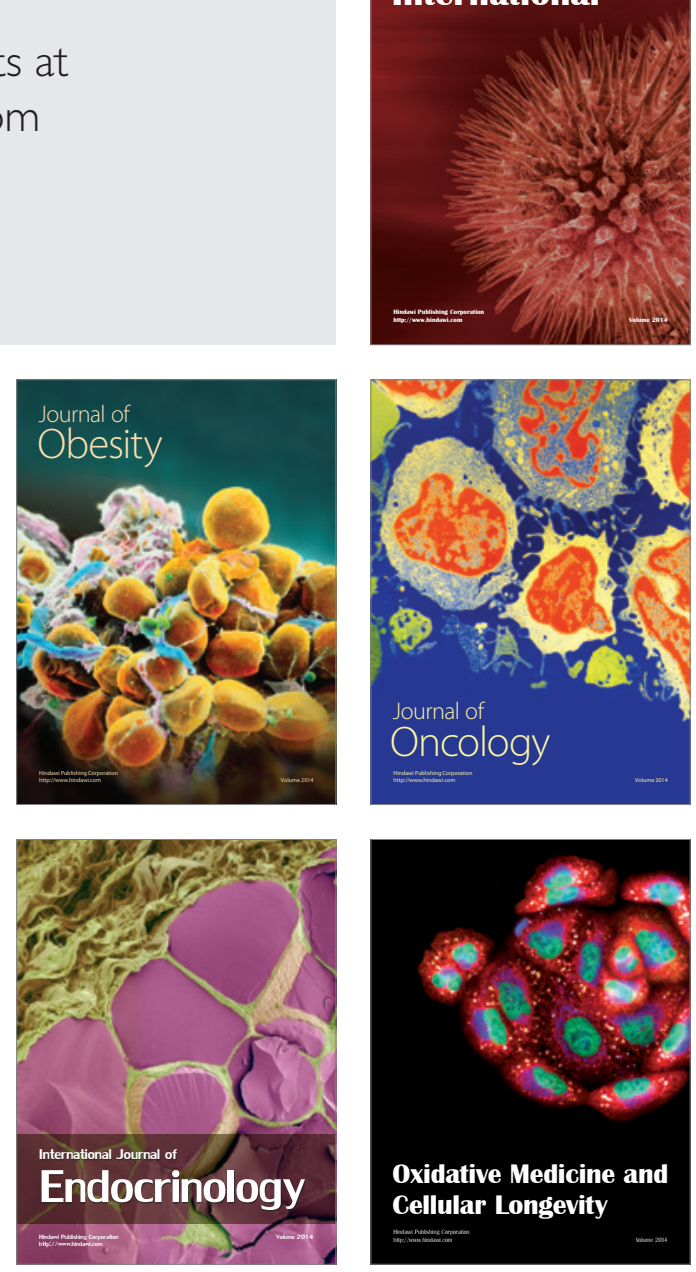